\title{
Elastic instability-mediated actuation by a supra-molecular polymer
}

\author{
Aviad Levin ${ }^{1,2 \dagger}$, Thomas C. T. Michaels ${ }^{2 \dagger}$, Lihi Adler-Abramovich ${ }^{3}$, Thomas O. Mason ${ }^{2}$, \\ Thomas Müller ${ }^{2,4}$, Bohan Zhang ${ }^{2}$, L. Mahadevan ${ }^{5,6}$, Ehud Gazit ${ }^{1,7 \star}$ and Tuomas P. J. Knowles ${ }^{2 \star}$
}

\begin{abstract}
In nature, fast, high-power-density actuation can be achieved through the release of stored elastic energy by exploiting mechanical instabilities in systems including the closure of the Venus flytrap' and the dispersal of plant or fungal spores ${ }^{2}$. Here, we use droplet microfluidics to tailor the geometry of a nanoscale self-assembling supra-molecular polymer to create a mechanical instability. We show that this strategy allows the build-up of elastic energy as a result of peptide selfassembly, and its release within milliseconds when the buckled geometry of the nanotube confined within microdroplets becomes unstable with respect to the straight form. These results overcome the inherent limitations of self-assembly for generating large-scale actuation on the sub-second timescale and illuminate the possibilities and performance limits of irreversible actuation by supra-molecular polymers.
\end{abstract}

Self-assembly is a ubiquitous phenomenon in nature that underlies the formation of the nanoscale machinery of life, including protein filaments $\mathrm{s}^{3,4}$, molecular motors ${ }^{5}$ and other complex architectures. This process involves the molecular recognition and association of building blocks mediated by non-covalent interactions, ultimately leading to supra-molecular species with unique characteristics, such as the ability to assemble reversibly, to modulate structure stiffness and to respond to external stimuli ${ }^{6-8}$. In biological systems, it has long been revealed that cellular movement and traction at surfaces is controlled by the self-assembly of cytoskeletal proteins $^{4,9-17}$.

The self-assembly of biomimetic building blocks is also an attractive route towards force generation in an artificial setting due to the fact that such processes take place under ambient conditions, with no, or minimal, requirements for external energy input. However, due to the highly dynamical nature of molecular-level self-assembly phenomena, it has been challenging to achieve rapid movement on length scales exceeding that of the building blocks themselves. A strategy to overcome these limitations is to decouple the slow build-up of potential energy, typically in the form of elastic energy, from its rapid release by exploiting mechanical instabilities. Natural systems use mechanical instabilities to generate remarkably rapid movements, including the closure of the Venus flytrap ${ }^{1}$ or the dispersal of spores and seeds by plants, fungi or bacteria ${ }^{2,18}$, but its coupling to self-assembly has not been reported. Here we focus on the dynamics of a dipeptide system, namely the self-assembly of diphenylalanine (FF) into nanostructures ${ }^{19-23}$. By confining the growing nanostructures inside microdroplets and presenting real-time imaging, we show that the self-assembly process can result in the build-up of elastic energy from the buckling and bending of the nanostructures.

To probe the force generated by the growth of self-assembled FF tubes, we used a microdroplet platform. FF was initially solubilized in acetic acid to form a stock solution of $100-300 \mathrm{mg} \mathrm{ml}^{-1}$ that was flowed directly into the microfluidic device; in a first junction on the device, this stock solution was diluted in equal part with water, yielding a super-saturated solution of $50-150 \mathrm{mg} \mathrm{ml}^{-1} \mathrm{FF}$. Immediately after mixing, this solution was compartmentalized into microdroplets at the second $\mathrm{T}$-junction on-chip into a fluorinated oil phase. Droplets were subsequently collected and stored off-chip.

We first observed the ability of FF building blocks (solution concentration $50 \mathrm{mg} \mathrm{ml}^{-1} \mathrm{FF}$ ) to self-assemble into robust tubes as a result of the partial evaporation of the aqueous/acetic acid phase when droplets were kept on a glass coverslide. An array of evaporating droplets in close proximity to each other was monitored over time, and we were able to detect that the formation of tubes in one droplet was accompanied by their expansion into other droplets in the vicinity of aggregated droplets (Fig. 1a). On closer inspection, nanotube formation inside droplets was observed to initiate from a single nucleation site from which tubes emerged and subsequently grew through secondary nucleation until they reached the droplet boundaries. Over time, the tubes were seen to penetrate through the membrane formed by the double interface and grow further inside neighbouring droplets (Fig. 1b).

We can quantify the forces implicated in FF self-assembly using classical nucleation theory. For a membrane formed by a thin oil layer between two aqueous droplets, exhibiting a surface tension $\sigma$, classical nucleation theory describes the free energy associated with the formation of a circular hole of radius $r$ as a balance between the cost of the formation of the hole and the gain in surface energy ${ }^{25}$ : $F(r)=2 \pi r \gamma-2 \pi r^{2} \sigma$, where $\gamma$ is the line tension (Fig. 1c). The competition between these two energy contributions leads to a free energy function $F(r)$ having a maximum as a function of hole radius (Fig. 1c). The critical radius and energy barrier emerge from this argument as: $r^{*}=\gamma /(2 \sigma)$ and $F^{*}=\pi \gamma^{2} /(2 \sigma)$. For our experimental values of $\sigma=20 \mathrm{pN} \mathrm{nm}^{-1}, \gamma=2 \mathrm{pN}$, we find $F^{*}=3.1 \times 10^{5} \mathrm{pN} \mathrm{nm}^{-1}$ (see Supplementary Information for details).

Linear FF assembly is thus able to generate forces that are sufficient to rupture water-in-oil double layer interfaces, but the rate of energy release is limited by the rate of FF self-assembly and

\footnotetext{
${ }^{1}$ Department for Molecular Microbiology and Biotechnology, Tel Aviv University, Tel Aviv 69978, Israel. ${ }^{2}$ Department of Chemistry, University of Cambridge, Lensfield Road, Cambridge CB2 1EW, UK. ${ }^{3}$ Department of Oral Biology, The Goldschleger School of Dental Medicine, Sackler Faculty of Medicine, Tel Aviv University, Tel Aviv 69978, Israel. ${ }^{4}$ Fluidic Analytics Ltd, Cambridge CB2 1EW, UK. ${ }^{5}$ School of Engineering and Applied Sciences, Harvard University, Cambridge, Massachusetts 02138, USA. ${ }^{6}$ Department of Physics, Harvard University, Cambridge, Massachusetts 02138, USA. ${ }^{7}$ Department of Materials Science and Engineering, Tel Aviv University, Tel Aviv 69978, Israel. ${ }^{\dagger}$ These authors contributed equally to this work. *e-mail: ehudg@post.tau.ac.il; tpjk2@cam.ac.uk
} 


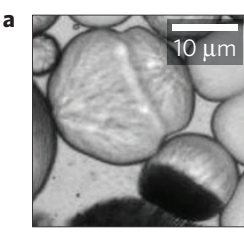

Os

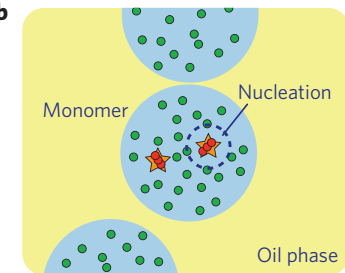

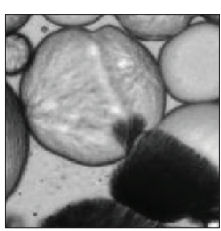

$11 \mathrm{~s}$

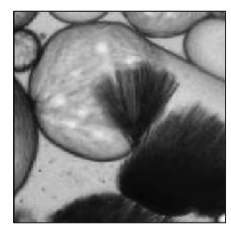

$40 \mathrm{~s}$

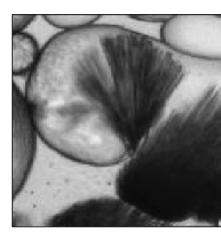

$86 \mathrm{~s}$
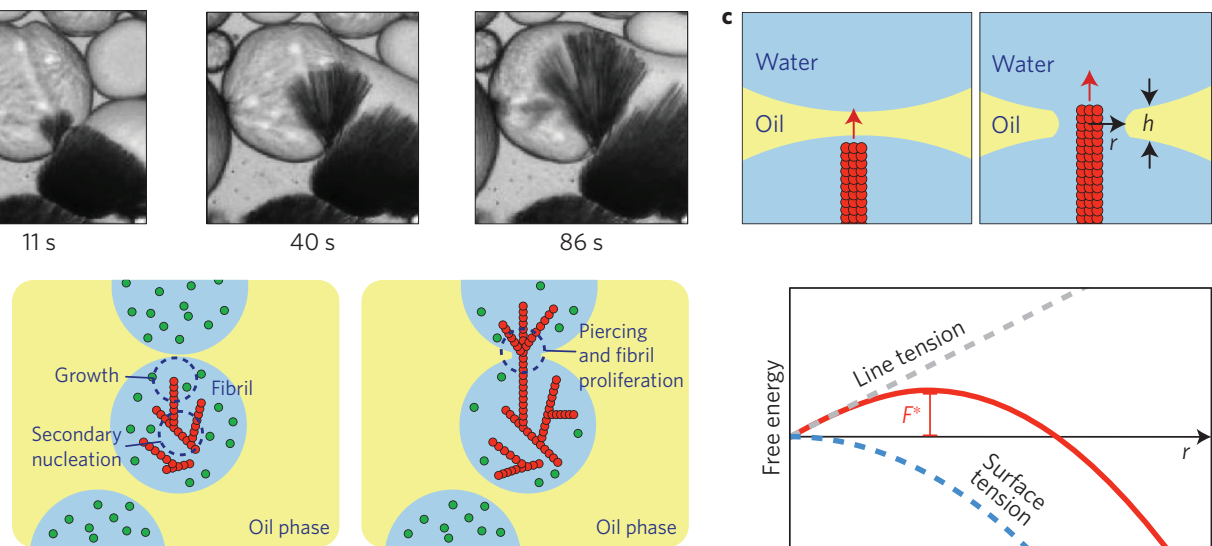

Figure 1 | FF tubes are able to pierce through water-in-oil double layer interfaces. a, Bright-field time-lapse microscopy of supercritical FF-containing

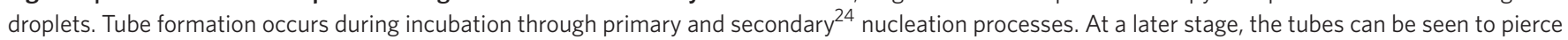
through the droplet interface, allowing the propagation of tube nucleation in surrounding droplets. $\mathbf{b}$, Schematic representation of the nucleation, growth

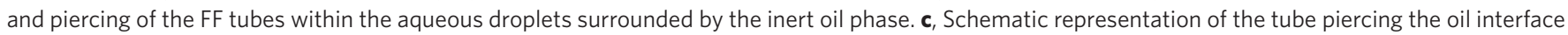
between adjacent droplets (top) and components involved in the calculation of the force generation (bottom).
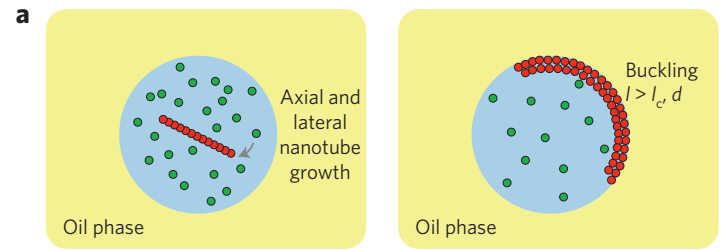

b

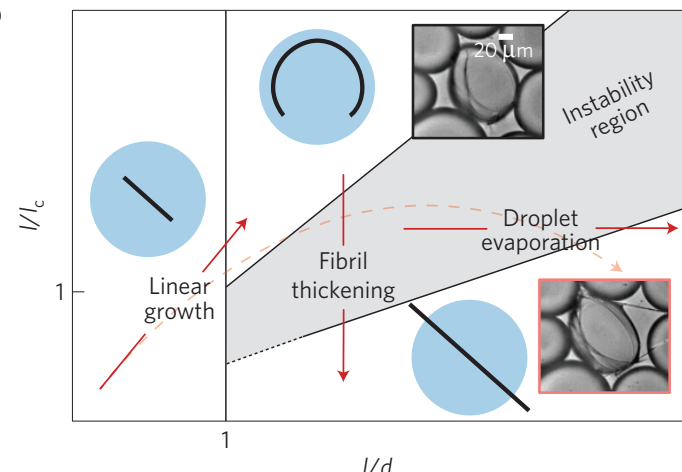

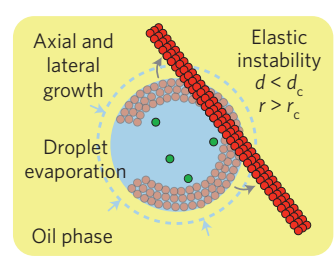

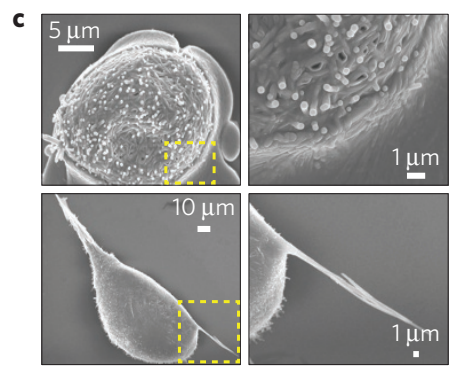

d

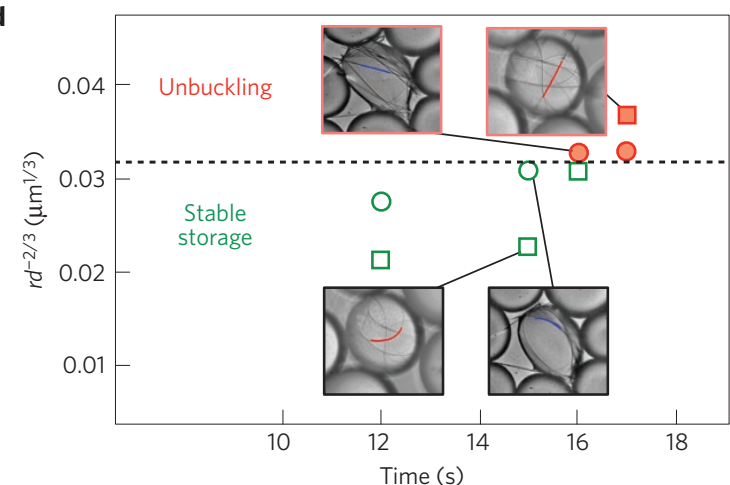

Figure 2 | Tailoring the geometry of FF self-assembly to create a mechanical instability. a, Schematics of fibril growth within supercritical droplets and their buckling. Fibrils may pierce through the droplet due to an increase in their cross-section, or through shrinkage of the droplet diameter. $\mathbf{b}$, Phase diagram displaying the relationship between tube length I, droplet diameter $d$ and Euler-buckling limit $I_{c}$. Panel adapted from ref. 26, PNAS. The solid arrows display the basic movement in phase space associated with linear tube growth, tube thickening and droplet evaporation. A typical trajectory in phase space, however, is a combination of these basic movements (dashed arrow). c, Scanning electron micrographs of dried droplets on a glass coverslip. While in the top droplet all tubes remain confined within the droplets because their cross-sectional radius is below the critical limit $r_{\mathrm{c}}$, in the bottom droplet several tubes with diameters over $1 \mu \mathrm{m}$ can be seen to emerge from the droplets. Insets show magnified regions of both droplets. $\mathbf{d}$, The growth of FF tubes inside individual droplets was followed using real-time imaging to determine the change in nanotube radius $r$ over time. The data indicate that below the unbuckling threshold, $r_{\mathrm{c}}$, nanotubes are stable with respect to straightening (green), whereas when $r$ exceeds $r_{\mathrm{c}}$ nanotubes unbuckle (red).

does not allow for large-scale actuation on sub-second timescales. We show here, however, that this limitation can be overcome by tailoring the geometry of the system to accumulate elastic energy through chemo-mechanical transduction and then release it rapidly as a result of an elastic instability. To this effect, we probed the self-assembly when initiated at $150 \mathrm{mg} \mathrm{ml}^{-1} \mathrm{FF}$ inside isolated microdroplets without the possibility for the structures to pierce through a thin oil layer into an adjacent aqueous compartment (see Supplementary Movie 1). Under such conditions, growing nanotubes undergo buckling when their length exceeds the Eulerbuckling limit $l_{\mathrm{c}}=(\pi E I /(2 r \sigma \cos \theta))^{1 / 2} \sim r^{3 / 2}$, with $E$ being the Young's modulus, $I=\pi r^{4} / 4$ the area moment of inertia of the nanotubes, $r$ the cross-sectional radius of filaments, $\sigma$ the surface tension of the oil/water interface and $\theta$ the contact angle (Fig. 2a, see Supplementary Information calculation for details) ${ }^{26}$. Further growth leads then to highly curved and strained structures that 


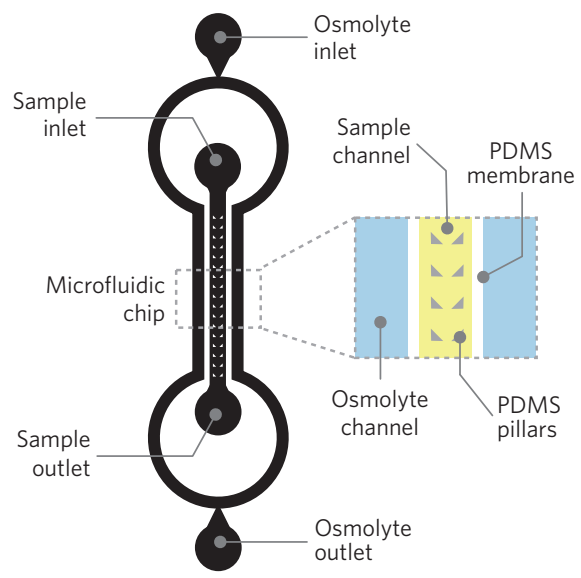

b

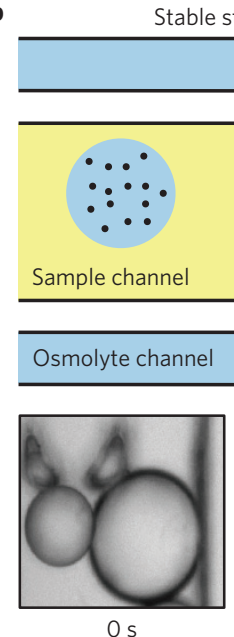

Stable storage

FC-40

PDMS membrane

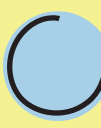

PDMS membrane

FC-40
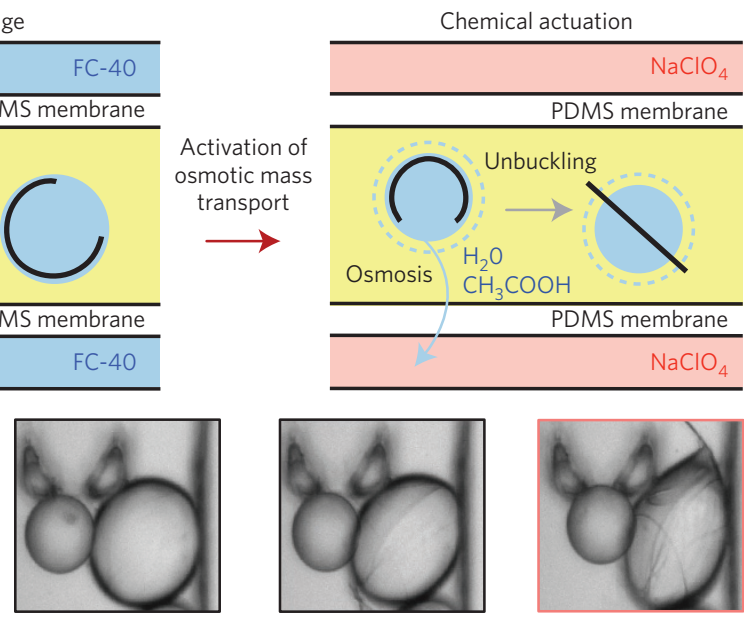

$10 \mathrm{~s}$

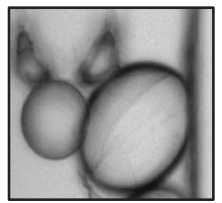

$20 \mathrm{~s}$

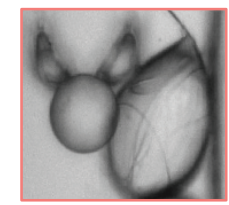

$30 \mathrm{~s}$

c
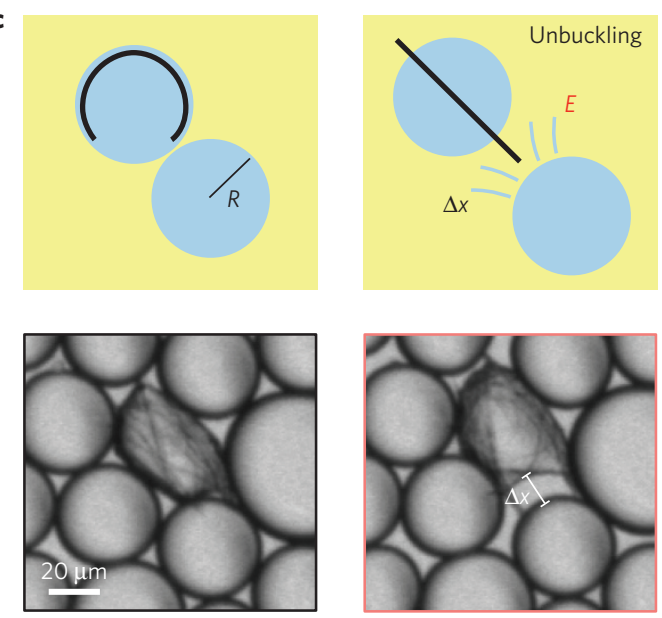

Figure $\mathbf{3}$ | Control of FF nanotube unbuckling by external stimuli and generation of external work. a, Schematics of the microfluidic device used for osmotic flow-driven actuation. $\mathbf{b}$, Bright-field time-lapse microscopy of FF tube self-assembly and buckling by altering the osmoticity of the surrounding solution. Osmotic diffusion of water from the droplets into 'sink' channels filled with $15 \mathrm{M} \mathrm{NaClO}_{4}$ solution separated from the main channel by thin-walled polydimethylsiloxane (PDMS) enabled tube actuation within droplets (right), while when FC-40 was used, no buckling could be detected (left).

c, Generation of external work by the unbuckling of FF nanotubes. The time interval between images was $10 \mathrm{~s}$.

follow the contour of the droplet interface. The elastic energy that is accumulated through the self-assembly of strained structures, $\mathcal{E}_{b}=2 E I / d^{2} l$ ( $d$ being the droplet diameter), is exactly compensated by the interfacial energy $\mathcal{E}_{i}=2 \pi r \sigma \cos \theta l$, as both contributions increase linearly with the length $l$ of FF nanotubes ${ }^{26}$. Thus, the release of the elastic energy cannot be achieved simply with nanotube elongation, but a detailed analysis of the mechanical stability of the system (see calculation in the Supplementary Information for details) reveals that the elastic energy effectively stored in the device can be released quickly from the system through a mechanical instability, with two modes of release being accessible: a decrease in droplet diameter below $d_{\mathrm{c}}=\sqrt{2} l_{\mathrm{c}} / \pi \approx 40 \mu \mathrm{m}$ through evaporation, or an increase in nanotube thickness, which can both lead to a situation where the buckled form of the nanostructure is unstable with respect to straightening (Fig. 2b). In the first case, removal of water and acetic acid from the droplet under conditions that favour evaporation (see Supplementary Methods for details) results in a decrease of the droplet diameter, and thus a higher bending energy of strained structures, compromising the balance between elastic and interfacial effects. Similarly, lateral growth of nanotubes, which is observed to take place in concomitance with growth in the axial direction (see Supplementary Movie 2 and Supplementary Fig. 1), can result in an unbalance between elastic and interfacial energies, as these increase with different dependencies on nanotube thickness. Theoretical predictions based on the material properties of FF nanotubes ${ }^{26,27}$ suggest that mechanical instability in a droplet of radius $70 \mu \mathrm{m}$ occurs once the crosssectional radius of the nanotubes exceeds $r_{\mathrm{c}}=\left(4 \sigma \cos \theta d^{2} / E\right)^{1 / 3} \sim$ $d^{2 / 3}$ ( $d$ being the droplet diameter) corresponding to about $500 \mathrm{~nm}$. Remarkably, high-resolution scanning electron microscopy imaging, in Fig. 2c, shows that the majority of the fibrils that remain contained within the droplets possess a radius of $500 \mathrm{~nm}$ or lower, while those that break through the droplet interface appear to have higher diameters of about $1 \mu \mathrm{m}$ or above. Indeed, following the tube actuation within a single droplet reveals that over a timescale of minutes, all tubes undergo unbuckling (see Supplementary Movie 3 and Supplementary Fig. 2). Furthermore, to investigate the dynamics of nanotube self-assembly and unbuckling, we have monitored the curvature and cross-sectional radius of individual tubes within the droplets until the buckling transition takes place (Supplementary Fig. 3). From the above theoretical considerations, a relevant combined parameter $r d^{-3 / 2}$ emerges that controls the system stability with respect to tube straightening. Remarkably, measurements of droplet diameters and nanotube cross-sectional radii show that the combined parameter $r d^{-3 / 2}$ increases as a function of time until reaching the critical value $r d^{-3 / 2}=(4 \sigma \cos \theta / E)^{1 / 3}$ at which point 


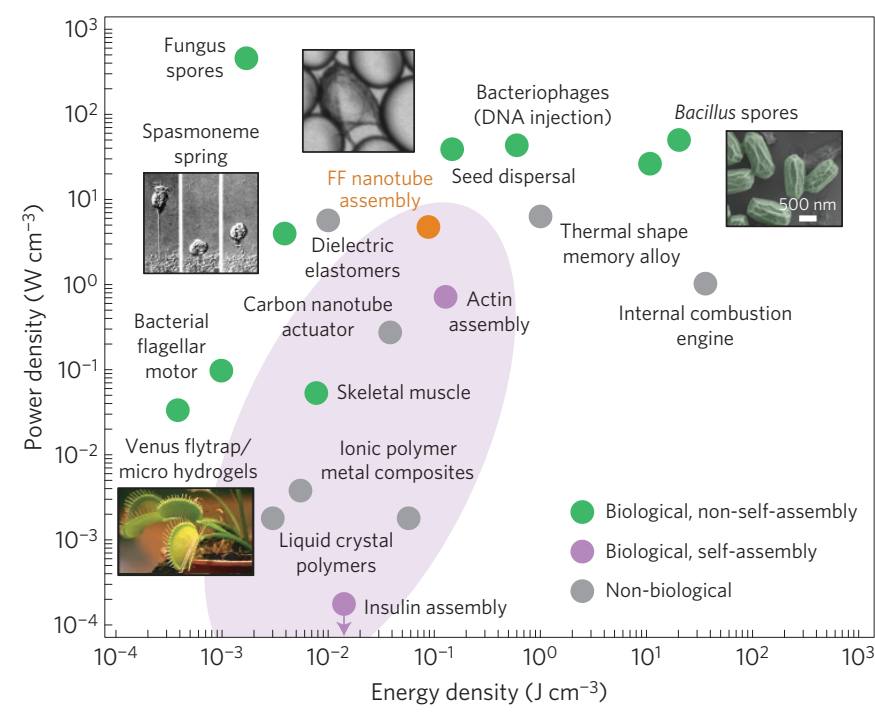

Figure 4 | Comparison of energy and power generation by biological and synthetic systems. Comparison of the chemo-mechanical transduction in FF nanotubes with energy $\left(\mathrm{J} \mathrm{cm}^{-3}\right)$ and power $\left(\mathrm{W} \mathrm{cm}^{-3}\right)$ densities generated by synthetic actuator systems (for example, shape memory alloys and internal combustion engines), biological stimuli-responsive materials (for example, seed and spore ejection systems) and biological self-assembly systems (for example, skeletal muscles). The purple shaded area represents the perimeter of energy and power density values reported for biological, self-assembling systems, as can be found in the ranges specified in Supplementary Table 1 and extended to the FF nanotube system. Images reproduced from: bottom left, ref. 1, NPG; top left, ref. 14, AAAS; top right, ref. 18, NPG.

the nanotubes are seen to undergo unbuckling, in accordance with our theoretical predictions (Fig. 2d).

We further explored the possibility to control the kinetics of unbuckling and subsequent power generation in our system by controlling the osmotic diffusion of water from the droplets into 'sink' channels. When the 'sink' channels are filled with a $15 \mathrm{M} \mathrm{NaClO}_{4}$ solution separated from the main channel by thinwalled polydimethylsiloxane (Fig. 3a) we were able to control the evaporation parameters (Fig. 3b). On the contrary, when the sink channels were filled with the surfactant FC-40, no selfassembly or buckling was detected even after about $10 \mathrm{~min}$ (data not shown). Alternatively, temperature can also be used to trigger tube unbuckling. Indeed, when supercritical droplets are incubated at $50{ }^{\circ} \mathrm{C}$ instead of $25^{\circ} \mathrm{C}$, the time required to observe the onset of the elastic instability becomes significantly shorter (Supplementary Fig. 4), demonstrating therefore the ability to exert precise control of instability-mediated chemo-mechanical transduction of nanotubes with an external stimulus. From a calculation of the bending energy accumulated in the droplet we could estimate the energy released from the unbuckling of a single nanotube to be $4 \times 10^{-12} \mathrm{~J}$, a value that corresponds to an energy density of $0.09 \mathrm{~J} \mathrm{~cm}^{-3}$ when normalized to the volume of the nanotubes (see calculation in the Supplementary Information for details). Using fast camera imaging, we determined an upper limit for the actuation time of $20 \mathrm{~ms}$, a value that results in a power density of $4.7 \mathrm{~W} \mathrm{~cm}^{-3}$. The measured value of the actuation time is in agreement with dimensionality arguments that suggest a time $t=\mu l^{3} / \mathcal{E}$ for the energy $\mathcal{E} \sim E r^{4} / l$ being released by motion of an object of a size $l$ in a liquid medium with viscosity $\mu$, giving $t=\mu l^{4} / E r^{4} \sim 6 \mathrm{~ms}$.

We also explored the possibility to generate external work using our actuating self-assembly system. To this effect, several droplets were brought into contact and their positions were monitored as an unbuckling transition took place within one of the droplets. We observed that the energy released from this unbuckling event was sufficient to physically displace the neighbouring droplets. We then estimated the amount of external work generated by the unbuckling of FF nanotubes, as illustrated in Fig. 3c, from the energy cost $\mathcal{E}=6 \pi \mu R\left(\Delta x^{2} / \Delta t\right) \sim 4 \times 10^{-13} \mathrm{~J}$ required to displace a droplet of radius $R \sim 20 \mu \mathrm{m}$ by a distance $\Delta x \sim 10 \mu \mathrm{m}$ over a timescale of $\Delta t \sim 10$ s, thus demonstrating the ability of our FF system to move micrometre-scale objects on second timescales.

In Fig. 4, we compare the results for the chemo-mechanical transduction in FF tubes obtained in the present study with literature values for the power generation by synthetic actuators, including shape memory alloys, biological self-assembly systems, and biological systems that exploit mechanical instabilities to release stored energy and accomplish important functional roles, including spore and seed ejection by plants and fungi. This comparison reveals that the energy and power densities provided by our FF system exceed those resulting from force generation by conventional self-assembly, including actin polymerization ${ }^{4,28}$. Thus, at a fundamental level, the exploitation of elastic instabilities has allowed us to generate a system that leverages biomimetic self-assembly to generate power densities that are higher than those reported in the past for biological self-assembly, and are similar to systems that were specifically designed to yield high energy release. The propensity of FF to self-assemble into ordered tubular structures was discovered over a decade ago ${ }^{29}$, and the robustness and rigidity of these tubes has been thoroughly studied ${ }^{30}$; in the present work, we have been able to enhance the power density of FF actuation through the exploitation of mechanical instabilities by coupling the nanoscale self-assembly reaction to micrometre-scale confinement. The real-time imaging analysis and modelling presented in this study establish elastic-instability actuation by supra-molecular polymers as a high-power-density mode of chemo-mechanical transduction and open up the possibility of using elastic instabilities for future applications in other short peptide, protein and synthetic polymer systems.

Data availability. The data that support the plots within this paper and other findings of this study are available from the corresponding author on request.

Received 26 April 2015; accepted 26 May 2016; published online 4 July 2016

\section{References}

1. Forterre, Y., Skotheim, J. M., Dumais, J. \& Mahadevan, L. How the Venus flytrap snaps. Nature 433, 421-425 (2005).

2. Noblin, X. et al. The fern sporangium: a unique catapult. Science 335, 1322 (2012).

3. Ideses, Y., Sonn-Segev, A., Roichman, Y. \& Bernheim-Groswasser, A. Myosin II does it all: assembly, remodeling, and disassembly of actin networks are governed by myosin II activity. Soft Matter 9, 7127-7137 (2013).

4. Higa, T., Suetsugu, N., Kong, S.-G. \& Wada, M. Actin-dependent plastid movement is required for motive force generation in directional nuclear movement in plants. Proc. Natl Acad. Sci. USA 111, 4327-4331 (2014).

5. Schliwa, M. \& Woehlke, G. Molecular motors. Nature 422, 759-765 (2003).

6. Brunsveld, L., Folmer, B., Meijer, E. \& Sijbesma, R. Supramolecular polymers. Chem. Rev. 101, 4071-4097 (2001).

7. Lehn, J. M. Perspectives in supramolecular chemistry- from molecular recognition towards molecular information processing and self-organization. Angew. Chem. Int. Ed. Engl. 29, 1304-1319 (1990).

8. Burnworth, M. et al. Optically healable supramolecular polymers. Nature 472, 334-338 (2011).

9. Ananthakrishnan, R. \& Ehrlicher, A. The forces behind cell movement. Int. J. Biol. Sci. 3, 303-317 (2007).

10. Aratyn-Schaus, Y., Oakes, P. W. \& Gardel, M. L. Dynamic and structural signatures of lamellar actomyosin force generation. Mol. Biol. Cell 22, 1330-1339 (2011).

11. Tan, J. L. et al. Cells lying on a bed of microneedles: an approach to isolate mechanical force. Proc. Natl Acad. Sci. USA 100, 1484-1489 (2003). 
12. Roberts, A. J., Kon, T., Knight, P. J., Sutoh, K. \& Burgess, S. A. Functions and mechanics of dynein motor proteins. Nature Rev. Mol. Cell Biol. 14, 713-726 (2013).

13. Granger, E., McNee, G., Allan, V. \& Woodman, P. The role of the cytoskeleton and molecular motors in endosomal dynamics. Semin. Cell. Dev. Biol. 31, 20-29 (2014).

14. Mahadevan, L. \& Matsudaira, P. Motility powered by supramolecular springs and ratchets. Science 288, 95-99 (2000)

15. Krause, M. \& Gautreau, A. Steering cell migration: lamellipodium dynamics and the regulation of directional persistence. Nature Rev. Mol. Cell Biol. 15, 577-590 (2014)

16. Amari, K., Di Donato, M., Dolja, V. V. \& Heinlein, M. Myosins VIII and XI play distinct roles in reproduction and transport of tobacco mosaic virus. PLoS Pathogens 10, el004448 (2014).

17. Utada, A. S. et al. Vibrio cholerae use pili and flagella synergistically to effect motility switching and conditional surface attachment. Nature Commun. 5, 4913 (2014).

18. Chen, X., Mahadevan, L., Driks, A. \& Sahin, O. Bacillus spores as building blocks for stimuli-responsive materials and nanogenerators. Nature Nanotech. 9, 137-141 (2014).

19. Sedman, V. L., Adler-Abramovich, L., Allen, S., Gazit, E. \& Tendler, S. J. B. Direct observation of the release of phenylalanine from diphenylalanine nanotubes. J. Am. Chem. Soc. 128, 6903-6908 (2006).

20. Hendler, N. et al. Formation of well-organized self-assembled films from peptide nanotubes. Adv. Mater. 19, 1485-1488 (2007).

21. Guo, C., Luo, Y., Zhou, R. \& Wei, G. Probing the self-assembly mechanism of diphenylalanine-based peptide nanovesicles and nanotubes. ACS Nano 6 , 3907-3918 (2012).

22. Kim, J. et al. Role of water in directing diphenylalanine assembly into nanotubes and nanowires. Adv. Mater. 22, 583-587 (2010).

23. Ikezoe, Y., Washino, G., Uemura, T., Kitagawa, S. \& Matsui, H. Autonomous motors of a metalorganic framework powered by reorganization of self-assembled peptides at interfaces. Nature Mater. 11, 1081-1085 (2012).

24. Hofrichter, J., Ross, P. D. \& Eaton, W. A. Kinetics and mechanism of deoxyhemoglobin S gelation: a new approach to understanding sickle cell disease. Proc. Natl Acad. Sci. USA 71, 4864-4868 (1974).

25. Kashchiev, D. Nucleation: Basic Theory with Applications (Oxford Univ. Press, 2000).
26. Cohen, A. E. \& Mahadevan, L. Kinks, rings, and rackets in filamentous structures. Proc. Natl Acad. Sci. USA 100, 12141-12146 (2003).

27. Kol, N. et al. Self-assembled peptide nanostructures are uniquely rigid bioinspired supramolecular structures. Nano Lett. 5, 1343-1346 (2005).

28. Hunter, I. \& Lafontaine, S. A comparison of muscle with artificial actuators Tech. Dig. IEEE Solid-State Sensor Actuator Workshop Hilton Head, South Carolina 178-185 (1992).

29. Reches, M. \& Gazit, E. Casting metal nanowires within discrete self-assembled peptide nanotubes. Science 300, 625-627 (2003).

30. Adler-Abramovich, L. et al. Thermal and chemical stability of diphenylalanine peptide nanotubes: implications for nanotechnological applications. Langmuir 22, 1313-1320 (2006)

\section{Acknowledgements}

This work was supported by a short-term fellowship from EMBO and from FEBS (A.L.), the Newman Foundation (A.L., T.O.M., T.P.J.K.), the Tel Aviv University Center for Nanoscience and Nanotechnology (A.L.), St John's College Cambridge (T.C.T.M.), the Israeli National Nanotechnology Initiative and Helmsley Charitable Trust (E.G.), Elan Pharmaceuticals (T.O.M.), the UK BBSRC (T.P.J.K.) and the ERC (T.P.J.K., T.C.T.M.). We thank P. Marcu and Z. Arnon for their assistance with the high-resolution scanning electron microscopy imaging, and members of the Gazit and Knowles groups for helpful discussion.

\section{Author contributions}

A.L., T.C.T.M., L.M., E.G. and T.P.J.K. conceived and designed the experiments. A.L., T.O.M., B.Z. and T.C.T.M. planned and performed the experiments. T.C.T.M., L.M. and T.P.J.K. developed the theory and analysed the experimental data. T.M. provided the fast camera set-up. A.L., T.C.T.M., L.A.-A., T.O.M., T.M., L.M., E.G. and T.P.J.K. wrote the manuscript. All authors discussed the results, provided intellectual input and critical feedback and commented on the manuscript.

\section{Additional information}

Supplementary information is available in the online version of the paper. Reprints and permissions information is available online at www.nature.com/reprints.

Correspondence and requests for materials should be addressed to E.D. or T.P.J.K.

\section{Competing financial interests}

The authors declare no competing financial interests. 